\title{
Directing the orientational alignment of anisotropic magnetic nanoparticles using dynamic magnetic fields
}

\author{
Daniel Hoffelner, ${ }^{a}$ Matthias Kundt, ${ }^{a}$ Annette M. Schmidt, ${ }^{a}$ \\ Emmanuel Kentzinger, ${ }^{b}$ Philipp Bender ${ }^{c}$ and Sabrina Disch ${ }^{\star a}$
}

Received 1st December 2014, Accepted 5th January 2015

DOI: $10.1039 / c 4 f d 00242 c$

The structure-directing influence of static and dynamic, i.e. rotating, magnetic fields on the orientational alignment of spindle-type hematite particles with a high aspect ratio is investigated. Structural characterization using electron microscopy and small-angle $X$-ray scattering confirms a nearly collinear particle arrangement with orientation of the main particle axis either parallel or perpendicular to the substrate as directed by the magnetic field geometry. The combination of large structural and magnetocrystalline anisotropies results in significantly different, strongly anisotropic magnetic properties of the assemblies revealed by directional magnetization measurements.

\section{Introduction}

The self-assembly of shape anisotropic nanoparticles into oriented arrangements is widely investigated due to its potential use for materials with anisotropic properties. ${ }^{\mathbf{1 - 4}}$ Faceted particles preferentially assemble with a face-to-face orientation, thus minimizing the van der Waals interaction energy between the nanoparticle facets. ${ }^{5-7}$ The mesoscopic assemblies of crystallographically aligned nanoparticles have been termed as mesocrystals. ${ }^{8}$ This emerging research field translates traditional crystallography into the mesoscale, with additional degrees of freedom induced by nanoparticle shape and orientation as compared to isotropic atoms.

Directional anisotropy is further accessible via field-induced self-assembly, where the directive forces exerted on nanoparticle building blocks by electric, ${ }^{9}$ magnetic, ${ }^{5,10}$ or flow fields ${ }^{11}$ are exploited towards oriented, long range ordered arrangements. The response of spherical magnetic nanoparticles to applied static and dynamic magnetic fields is the subject of intense research in view of its

${ }^{a}$ Department Chemie, Institut für Physikalische Chemie, Universität zu Köln, Luxemburger Str. 116, D-50939 Köln, Germany. E-mail: sabrina.disch@uni-koeln.de

${ }^{b}$ Jülich Centre for Neutron Science JCNS, Peter Grünberg Institut PGI, JARA-FIT, Forschungszentrum Jülich GmbH, D-52425 Jülich, Germany

${ }^{c}$ Experimentalphysik, Universität des Saarlandes, Postfach 151150, D-66041 Saarbrücken, Germany 
fundamental technological importance, e.g. for medical applications such as imaging and magnetic hyperthermia, ${ }^{12}$ or sensor applications. ${ }^{13}$ For macroscopic spherical particle dispersions, a variety of complex structures has been obtained via self-assembly techniques driven by time-dependent magnetic fields. ${ }^{\mathbf{1 4}}$

The combination of the structure-directing influence of static or dynamic magnetic fields with the structural anisotropy of elongated nanoparticles represents a promising approach towards directional alignment of magnetic nanoparticles, aiming at nanostructures with directionally anisotropic magnetic properties. Suitable model systems for this approach include hematite particles of spindle-type shape due to their perpendicular structural and magnetic main axes. Hematite nanospindles are routinely synthesized with orientation of the crystal$\operatorname{lographic} c$ axis of the hematite corundum structure along the main rotation axis of the spindle. ${ }^{15,16}$ Above the Morin temperature at $T \sim 263 \mathrm{~K}$, the material exhibits weakly ferromagnetic behavior resulting from a slight canting of the antiferromagnetic sublattices. Due to the magnetocrystalline anisotropy, the small net magnetization vector is oriented in the basal plane of the material, whereas additional contributions from uncompensated spins are possible for smaller particle sizes. ${ }^{17}$ In consequence, hematite nanospindles in aqueous dispersion preferentially orient with their long axis perpendicular to an applied magnetic field, ${ }^{18,19}$ reducing the degrees of freedom for particle orientation.

Here, we present the magnetic field-directed self-organization of hematite spindles into oriented nanoparticle assemblies. By application of static and dynamic magnetic fields during the evaporation-mediated self-assembly process, nearly collinear particle arrangements are achieved. Uniaxial alignment of the particle assemblies either parallel or perpendicular to the substrate is tuned by the application of static and rotating magnetic fields, respectively. The strongly anisotropic magnetic properties arising from the orientational alignment in the obtained assemblies are studied using directional magnetization measurements.

\section{Experimental}

\subsection{Nanoparticle synthesis and self-assembly}

Sodium dihydrogenphosphate monohydrate $\left(\mathrm{NaH}_{2} \mathrm{PO}_{4} \cdot \mathrm{H}_{2} \mathrm{O}\right.$, p.a.) was obtained from Applichem. Tetramethylammonium hydroxide (TMAOH, 25\% in water) was obtained from Sigma-Aldrich. Iron(III) chloride hexahydrate (98\%, ABCR GmbH) and citric acid monohydrate (p.a. quality, Jungbunzlauer $\mathrm{GmbH}$ ) were used without further purification. The water used for sample preparation and analysis was obtained from Acros Organics in analytical quality.

The synthesis of spindle-like hematite $\left(\alpha-\mathrm{Fe}_{2} \mathrm{O}_{3}\right)$ nanoparticles is based on the thermolysis of aqueous ferric chloride $\left(\mathrm{FeCl}_{3}, c=0.02 \mathrm{~mol} \mathrm{~L}^{-1}\right)$ in the presence of $\mathrm{NaH}_{2} \mathrm{PO}_{4}\left(c=0.54 \times 10^{-3} \mathrm{~mol} \mathrm{~L} \mathrm{~L}^{-1}\right)$ initially reported by Matijevic and coworkers. ${ }^{15}$ In the present synthesis, the starting materials were combined after pre-heating to $90{ }^{\circ} \mathrm{C}$ and thermolized at $100{ }^{\circ} \mathrm{C}$ for 96 hours. After thorough washing, the particles were electrostatically stabilized with citric acid and TMAOH in water. ${ }^{16}$

Self-assembly of the nanoparticles was performed by depositing a defined amount of the nanoparticle dispersion on a pre-cleaned $10 \times 10 \mathrm{~mm}^{2}$ silicon substrate in three different magnetic environments. For the zero field reference, this drop casting process was carried out using $75 \mu \mathrm{L}$ of a $3.3 \mathrm{vol} \%$ dispersion in 
the absence of a magnetic field. For self-assembly in a static magnetic field, $50 \mu \mathrm{L}$ of a $3.3 \mathrm{vol} \%$ dispersion was deposited, and a static magnetic field of $\mu_{0} H=38 \mathrm{mT}$ was applied horizontally, i.e. parallel to the substrate, during evaporation. For self-assembly in dynamic magnetic fields, a rotating magnetic field in the substrate plane was generated by two crossed sets of Helmholtz coils providing AC magnetic fields, operated with a relative phase shift of $\pi / 2$. Using this geometry, $75 \mu \mathrm{L}$ of a 1.0 vol\% dispersion was deposited in a rotating magnetic field of $\mu_{0} H=20 \mathrm{mT}$ and a frequency of $200 \mathrm{~Hz}$.

\subsection{Characterization}

2.2.1 Electron microscopy. Scanning Electron Microscopy (SEM) images were obtained using a Zeiss Neon 40 microscope operated at $5 \mathrm{kV}$. For determination of the particle morphology and size distribution, a drop of dilute nanoparticle dispersion was placed on a silicon substrate and evaporated. Particle size histograms were obtained by manual measurement of diameter, length, and aspect ratio of more than 100 particles. The mean size and size distribution were obtained by fitting the corresponding histogram with a lognormal distribution function. The nanoparticle assemblies on silicon substrates were sputtered with a gold layer of $\sim 10 \mathrm{~nm}$ thickness before imaging.

2.2.2 Dynamic light scattering. DLS measurements were carried out using a laboratory built goniometer equipped with an $\operatorname{Ar}+\operatorname{laser}(\lambda=488 \mathrm{~nm})$, fiber optical detector and ALV autocorrelator. The scattering cells ( $10 \mathrm{~mm}$ cylindrical cuvettes, VWR) were immersed in a thermostated index matching bath (toluene). All measurements were carried out at $25{ }^{\circ} \mathrm{C}$ and the temperature was adjusted to an absolute accuracy of $0.1{ }^{\circ} \mathrm{C}$. The typical laser power was $15 \mathrm{~mW}$. For depolarized dynamic light scattering measurements, the primary beam and the scattered light passed through Glan-Thompson polarizers.

2.2.3 Magnetization measurements. Quasistatic magnetization measurements were carried out on an ADE Magnetics Vibrating Sample Magnetometer (VSM) EV7. Field-dependent magnetization data was collected in a field range of $\pm 2.2 \mathrm{~T}$ at $298 \mathrm{~K}$ with a head drive frequency of $75 \mathrm{~Hz}$. For measurements of the dilute nanoparticle dispersion, the sample was sealed in a Teflon crucible and placed on a glass sample holder. For directionally dependent magnetization measurements of nanoparticle assemblies, the sample was directly attached to the glass sample holder and oriented with respect to the poles of the electromagnet. All measurements were carefully corrected for the diamagnetic magnetization contribution originating in the sample holder and blank silicon substrate.

AC magnetization measurements were carried out on an Imego DynoMag Susceptometer. An alternating magnetic field of $\mu_{0} H=0.5 \mathrm{mT}$ in a frequency range of $0.1 \mathrm{~Hz}-10 \mathrm{kHz}$ was applied during measurement of the real and imaginary parts of the magnetic susceptibility.

2.2.4 Small-angle X-ray scattering. SAXS and surface-near SAXS measurements were carried out at the high brilliance laboratory Gallium Anode Low Angle X-ray Instrument GALAXI at the research center Jülich (Germany). The instrument is based on the former B1 beamline of the DORIS synchrotron at HASYLAB (Deutsches Elektronen Synchrotron DESY, Hamburg, Germany) and now equipped with a Bruker AXS MetalJet X-ray source providing highly intense $\left(10^{9} \mathrm{~s}^{-1}\right)$ 
X-rays with a wavelength of $1.34 \AA$ A. The scattering intensity was recorded on a $1 \mathrm{M}$ Pilatus detector (DECTRIS) positioned at a distance to the sample of $3.526 \mathrm{~m}$. For SAXS measurements of the nanoparticle form factor, a dilute aqueous dispersion of the particles was sealed in a $1.5 \mathrm{~mm}$ quartz capillary, and the measured data was averaged circularly and corrected for solvent scattering for evaluation. As the accessible range of the scattering wave vector $Q$ does not allow for refinement of the entire particle form factor with its large main axis, the obtained data is compared to simulations based on the particle morphology obtained from SEM characterization. Surface-near SAXS measurements by nanoparticle assemblies deposited on silicon substrates were carried out with the sample aligned horizontally in the direct beam. Incident angles of $0.3-0.5^{\circ}$ were chosen. The high thickness and roughness of the deposited particle layer results in a smeared parasitic scattering intensity in the vertical $\left(Q_{z}\right)$ direction, which was masked for analysis. The form factor of linearly oriented ellipsoidal particles was simulated using the SasView software.

\section{Results \& discussion}

\subsection{Particle synthesis and characterization}

Elongated hematite nanoparticles were prepared with narrow size distribution according to established synthesis procedures. ${ }^{15,16}$ Morphology and physical properties of the obtained particles were characterized using dilute, i.e. noninteracting dispersions. Scanning electron microscopy (SEM) images presented in Fig. 1a confirm the uniform spindle-type particle shape with particle diameter and length of $65(1) \mathrm{nm}$ and $447(7) \mathrm{nm}$, respectively, along with a lognormal particle size distribution of $\sigma_{\log }=15 \%$ in all dimensions and a high aspect ratio of 6.6(1). Small-angle X-ray scattering (SAXS) data by nanoparticles in dilute aqueous dispersion (Fig. 1a) is in agreement with an ellipsoidal particle form factor ${ }^{20,21}$ corresponding to the particle morphology determined using SEM and confirms the determined particle size distribution for large sample statistics.

DC magnetization measurements of the particles in aqueous dispersion (Fig. 1c) exhibit the pseudo-superparamagnetic Langevin-type behavior induced by Brownian rotation of the weakly ferromagnetic spindles. Beyond reaching the spontaneous magnetization $\sigma_{\mathrm{s}}$, excess susceptibility is observed at high magnetic field, as is known for hematite. ${ }^{17}$ The magnetization data in Fig. 1c has been corrected for this excess susceptibility term and fitted according to the Langevintype behavior scaled by the spontaneous magnetization

$$
\sigma_{\mathrm{s}} \mathscr{L}(\xi)=\sigma_{\mathrm{s}}\left[\operatorname{coth}(\xi)-\frac{1}{\xi}\right],
$$

with the Langevin parameter

$$
\xi=\frac{\mu \mu_{0} H}{k_{\mathrm{B}} T}
$$

where $\mu$ is the integral particle moment, $\mu_{0} H$ the applied field, $k_{\mathrm{B}}$ the Boltzmann constant, and $T$ the temperature. The obtained spontaneous magnetization of $\sigma_{\mathrm{s}}=1187(3) \mathrm{A} \mathrm{m}^{-1}$ represents only $58 \%$ of the bulk value for hematite of $2000 \mathrm{~A} \mathrm{~m}^{-1},{ }^{17,22}$ in agreement with earlier reports. ${ }^{23}$ The integral nanoparticle moment of $\mu=1.19(2) \times 10^{-18} \mathrm{~J} \mathrm{~T}^{-1}$ corresponds to a magnetic particle volume of 

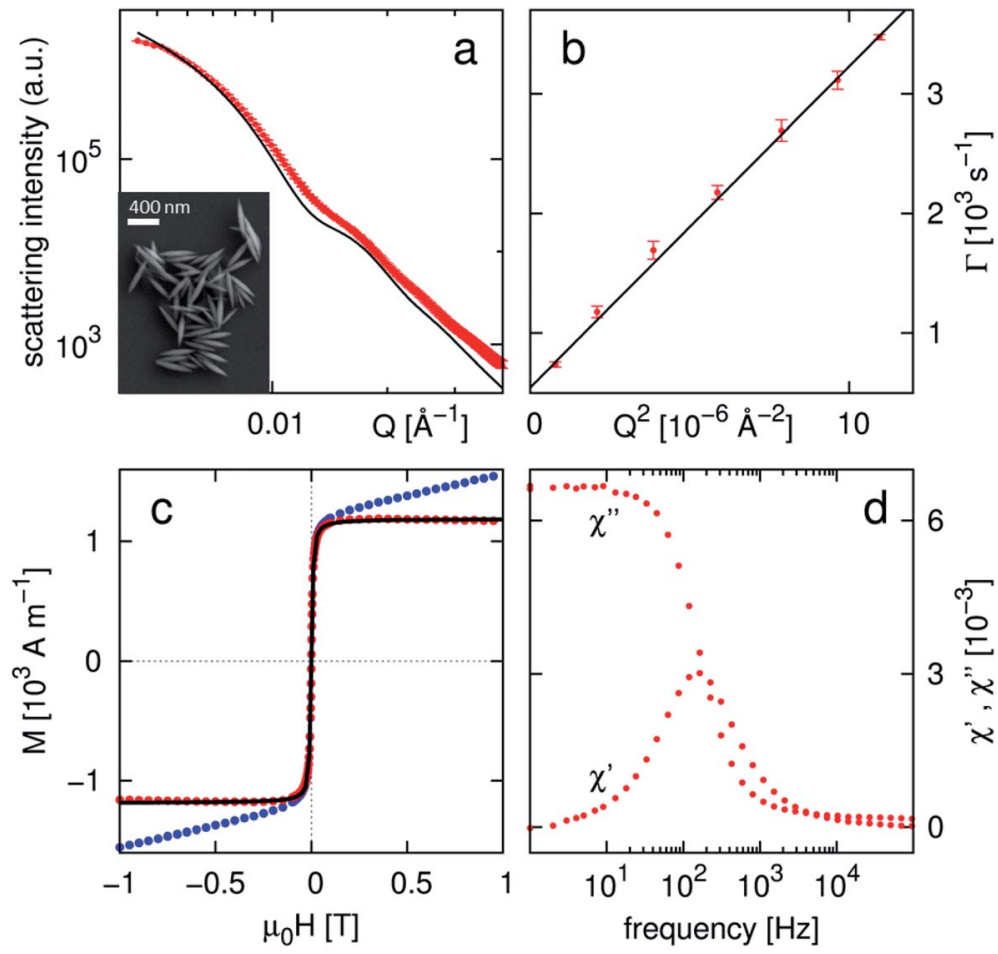

Fig. 1 Morphological, magnetic, and dynamic characterization of the hematite nanoparticles under study (a) SAXS data (red) with ellipsoidal form factor simulation (black), typical SEM images, (b) depolarized DLS data, (c) magnetization by nanoparticles in dispersion as measured (blue), after correction of $\chi_{\text {exc }}$ at high fields (red), with Langevin fit (black), (d) AC susceptibility data.

$V_{\mathrm{m}}=\mu / \sigma_{\mathrm{s}}=1.01(2) \times 10^{-21} \mathrm{~m}^{3}$, in excellent agreement with the morphological particle volume $V_{\mathrm{p}}=0.99 \times 10^{-21} \mathrm{~m}^{3}$, as determined from SEM and SAXS analysis. The dynamical behavior of the spindles in aqueous dispersion was investigated using dynamic light scattering (DLS, Fig. 1b) as well as AC magnetometry (Fig. 1d). Angle-dependent DLS measurements yield a translational diffusion coefficient $D_{\mathrm{t}}$ of $2.98(4) \times 10^{-12} \mathrm{~m}^{2} \mathrm{~s}^{-1}$. Using depolarized DLS measurements, a rotational diffusion coefficient of $D_{\mathrm{r}}=91(4) \mathrm{s}^{-1}$ is determined. The good agreement with theoretical values calculated for ellipsoidal particles $\left(D_{\mathrm{t}}=2.85 \times 10^{-12} \mathrm{~m}^{2} \mathrm{~s}^{-1}\right.$, $\left.D_{\mathrm{r}}=103 \mathrm{~s}^{-1}\right)^{24}$ emphasizes again the uniform particle shape. The rotational diffusion coefficient determined here is associated with the barrier for rotation around the minor spindle axis in the applied conditions of temperature (RT) and carrier liquid (water) viscosity and relates to a characteristic frequency of $\nu_{\mathrm{c}}=D_{\mathrm{r}} / \pi=29(1) \mathrm{Hz}^{25}$ AC susceptibility measurements are sensitive to a characteristic frequency resulting from a superposition of the barriers for rotation around the minor and major spindle axes. Consequently, the characteristic frequency of $\nu=160 \mathrm{~Hz}$ determined here is significantly larger than that obtained from depolarized DLS, and comparison of both values indicates a characteristic frequency of rotation around the major spindle axis beyond $200 \mathrm{~Hz}$. 
Overall, the prepared nanospindles thus exhibit a suitable uniformity in size and shape for self-organization experiments, and the dynamic reorientation behavior characterized by depolarized DLS and AC-susceptibility measurements indicates a suitable frequency window for dynamic field induced self-assembly experiments.

\subsection{Self-organization in static magnetic field}

Drop-casting dispersions of the prepared hematite particles yields assemblies with a varying degree of orientational order that is significantly enhanced and directed by application of a magnetic field. Representative SEM images of typical assemblies obtained without magnetic field and with application of a static magnetic field of $38 \mathrm{mT}$ parallel to the substrate are presented in Fig. 2a and b, respectively. In the absence of a directing magnetic field, only weak, local correlations are observed. Side-to-side orientation of the particles is likely induced by van der Waals interactions along the particles resulting from the elongated particle shape, leading to small domains of $\sim 5-10$ particles in a locally collinear arrangement. The strongly disordered orientation of these domains, however, amounts to a macroscopically isotropic sample.

Application of a static magnetic field during self-assembly induces parallel alignment of the spindle-shaped particles similar to the nematic phase of liquid crystalline materials. The observed orientation of the long particle axis perpendicular to the inducing magnetic field direction (Fig. 2b) is in agreement with the known orientation of the easy axis of the material in the basal plane of the spindles. ${ }^{18,19}$ The top view of electron microscopy techniques indicates such a collinear arrangement on the entire sample surface, but does not give information on the particle organization in the interior of the assembly. Considering the large thickness of the assembled material in the order of $20 \mu \mathrm{m}$, the potential configurations in agreement with the SEM observations consist of either collinear

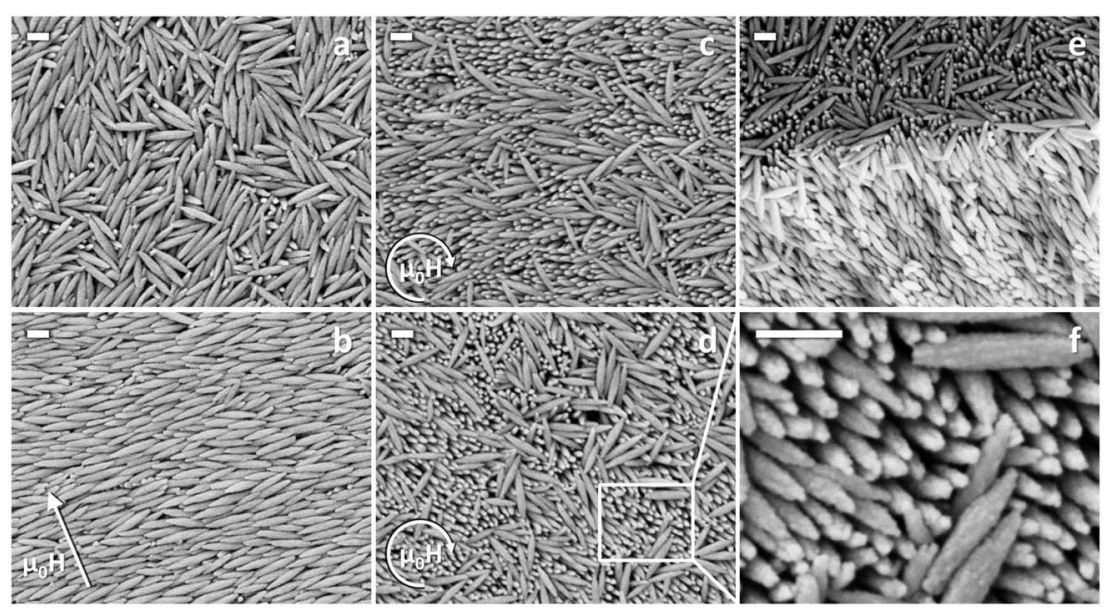

Fig. 2 SEM images of hematite nanospindles assembled in (a) absence of a magnetic field, (b) static field of $38 \mathrm{mT}$ in the indicated direction, (c and d) rotating magnetic field of 200 $\mathrm{Hz}$ and $20 \mathrm{mT}$, along with (e) side view of a breaking edge of the sample assembled in a rotating field and (f) detailed view. Scale bars represent $200 \mathrm{~nm}$. 
arrangement or orientational disorder in the plane perpendicular to the inducing magnetic field in the interior of the assembly.

In order to get insight into the particle orientation in all dimensions throughout the sample, SAXS measurements were carried out on the completely dried nanospindle assembly shown in Fig. 2b. The sample was oriented with the direction of the magnetic field applied during self-assembly either parallel (Fig. 3a) or perpendicular (Fig. 3d) to the incident X-ray beam. The 2D SAXS data presented in Fig. 3a and $d$ is in a first approximation interpreted by comparison with the simulated orientation distribution of an ellipsoidal form factor (Fig. 3b and e). The scattering intensity distribution in Fig. $3 \mathrm{a}$ is in excellent agreement with horizontal orientation of the ellipsoidal form factor with the long particle axis perpendicular to the incident X-ray beam (Fig. 3b), whereas the scattering intensities collected after $90^{\circ}$ rotation of the sample (Fig. $3 \mathrm{~d}$ ) correspond well to the isotropic intensity distribution expected for orientation of ellipsoidal particles with their long particle axis parallel to the X-ray beam (Fig. 3e). In both cases the ellipsoidal form factor has been simulated with an orientation distribution of $5^{\circ}$. These observations confirm a nearly collinear arrangement of the particles throughout the sample. We attribute the collinear particle orientation in our deposited samples, in contrast to the 2D disordered orientation found in dispersion, ${ }^{18}$ to steric effects such as van der Waals interactions between the particles or excluded volume effects, ${ }^{26}$ which become more important with increasing particle concentration upon evaporation. The horizontal orientation of the nematic phase is likely induced by the flat geometry of the substrate. However, the observation of isotropic particle orientation in the absence of a magnetic field

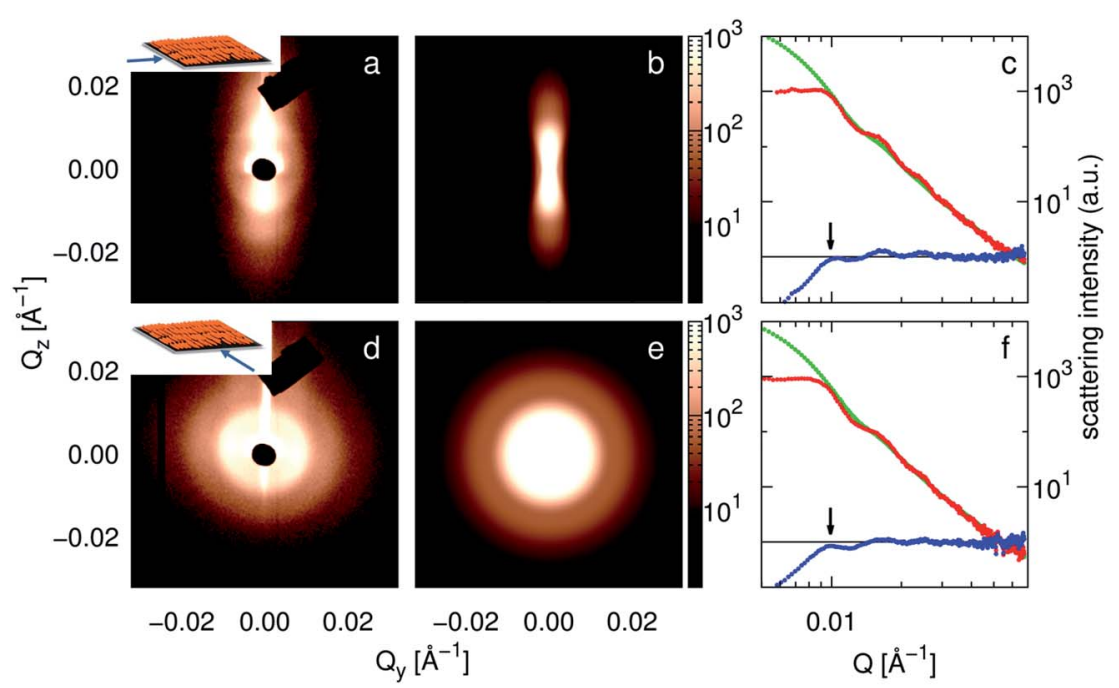

Fig. 3 Small-angle $X$-ray scattering by nanospindle assemblies deposited in a static magnetic field. Sample orientation with the direction of the applied field during deposition (a) parallel and (d) perpendicular to the incident X-ray beam (inset). (b and e) Simulated orientation distribution of the ellipsoidal form factor. ( $c$ and f) Extraction of the structure factor observed in (a) and (d), respectively. Red: integrated scattering intensity in a $20^{\circ}$ sector in $Q_{z}$ (c) and $Q_{y}$ (f) directions, green: scaled SAXS form factor as measured in dispersion, blue: extracted structure factor with marked correlation maximum. 
(Fig. 2a) illustrates that steric effects alone are not sufficient to induce collinear alignment, thus emphasizing the strong orientation-directing effect of a magnetic field during particle deposition. The most significant difference between data and form factor $(P(Q))$ simulations is a pronounced modulation of the experimental scattering intensities $(I(Q))$, which is associated with the structure factor $(S(Q))$ of the densely packed particles according to

$$
I(Q)=P(Q) \cdot S(Q)
$$

where $Q$ is the magnitude of the scattering wave vector related to the scattering angle $2 \theta$ and the X-ray wavelength $\lambda$ by $Q=\frac{4 \pi}{\lambda} \sin (\theta)$. Analysis of the scattering intensities in Fig. 3a and d perpendicular to the long particle axis in the vertical and horizontal directions, respectively, reveals a weak structure factor (Fig. 3c and f), with a correlation maximum at $Q=0.0099(2) \AA^{-1}$ for both directions. The weakness of observed structural correlation is attributed to the short range order of the particle organization similar to nematic phases without the long structural coherence of crystalline particle arrangements. A long range ordered crystalline arrangement, associated with a much stronger structure factor and resulting in sharp Bragg peaks, requires nanoparticles of significantly narrower size distribution. ${ }^{6}$ The determined correlation maximum corresponds to a real space correlation distance of 635(13) $\AA$, which is in excellent agreement with the particle diameter, thus confirming the densely packed particle arrangement observed by SEM for a statistically significant part of the sample. The combination of electron microscopy and X-ray scattering thus reveals the sample organized in a static magnetic field as a good example for a collinearly arranged ensemble of elongated hematite particles oriented parallel to the underlying substrate.

\subsection{Self-organization in rotating magnetic field}

Extension of the field induced self-assembly technique towards dynamic magnetic fields provides a way to employ the different rotational diffusion coefficients of the major and minor particle axes towards collinear arrangements of hematite spindles with orientation perpendicular to the substrate. In a magnetic field rotating in between these characteristic frequencies, rotation of the spindles around their major axis is energetically favorable and is thus expected to yield perpendicularly oriented assemblies upon drop casting.

Deposition of the hematite particles in a magnetic field of $\mu_{0} H=20 \mathrm{mT}$ rotating in the substrate plane with $200 \mathrm{~Hz}$ indeed yields particle arrangements with preferred orientation nearly perpendicular to the substrate. Representative SEM images presented in Fig. 2c-f reveal a large number of particles that are collinearly arranged, but covered with a surface layer of disordered particles. The disordered surface layer can be understood as a result of particle alignment within the air/liquid droplet surface upon deposition of the sample, thereby reducing the high surface tension of the dispersion. ${ }^{27}$ In consequence, a disordered particle layer remains on top of the prepared assemblies upon final evaporation of the droplet. Below this surface layer of disordered spindles, SEM images reveal a collinear arrangement of particles with orientation nearly perpendicular to the substrate. A slight inclination of the particle orientation with respect to the substrate normal is observed (Fig. $2 c$ and d). A tilted view of a 
breaking edge of the sample (Fig. 2e) indicates that the observed collinear, perpendicular spindle orientation ranges far into the interior of the assembly.

SAXS measurements carried out at different positions of one and the same sample confirm the collinear spindle orientation on a larger scale and give detailed information on the orientation with respect to the substrate. The $2 \mathrm{D}$ scattering intensity presented in Fig. $4 \mathrm{a}-\mathrm{c}$ is again well represented by an oriented ellipsoidal form factor (simulated in Fig. $4 \mathrm{~d}-\mathrm{f}$ with $5^{\circ}$ orientation distribution), modulated with the structure factor already discussed in 3.2. Here, varying orientations of the ellipsoidal form factor with inclination angles of $0^{\circ}$ to $35^{\circ}$ between the major particle axis and the $Q_{z}$ direction are found on different locations on the sample. The precise inclination angles observed with only a small orientation distribution indicate the presence of large domains with nearly collinear particle orientation in a constant inclination angle and direction, as opposed to a powder-like distribution of inclination directions in the sample. This is in agreement with SEM observations, where a different inclination direction of the particle orientation is found on different locations of the sample (Fig. 2c and d), whereas the sample had not been rotated but only laterally shifted between the two images. Given that at most 2-3 different domains of inclination direction are observed by SAXS (Fig. 4c), the domain size is estimated to be close to the order of magnitude of the X-ray beam footprint on the sample $\left(1 \times 10 \mathrm{~mm}^{2}\right)$. For a quantitative discussion of the inclination angle, it has to be considered that the measured 2D scattering intensity corresponds to a projection of the $3 \mathrm{D}$ form factor onto the detector plane. Consequently, the true particle inclination angle may be slightly larger than the observed inclination on the $2 \mathrm{D}$ detector. The observed large inclination angles of up to $35^{\circ}$ are attributed to the relatively low

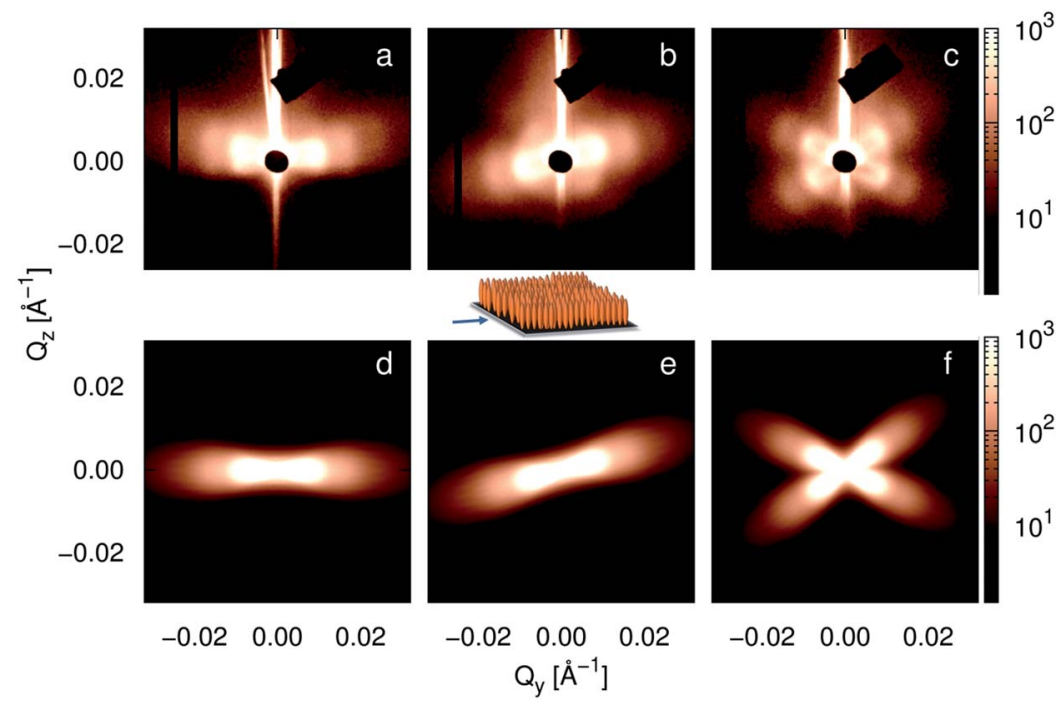

Fig. 4 Small-angle $X$-ray scattering by nanospindle assemblies deposited in a rotating magnetic field. SAXS data obtained at different positions on the same sample is presented $(\mathrm{a}-\mathrm{c})$, along with simulations of the orientation distribution of the ellipsoidal form factor corresponding to (d) perpendicular orientation of the spindles on the substrate and tilted at (e) $15^{\circ}$ and (f) $35^{\circ}$ and $-25^{\circ}$ to the domains of nanospindles. Orientation of the sample in the $\mathrm{X}$-ray beam is depicted in the center. 
magnitude of the inducing magnetic field. The Langevin parameter for the studied particles $\left(\mu=1.19(1) \times 10^{-18} \mathrm{~J} \mathrm{~T}^{-1}\right)$ and field strength $\left(\mu_{0} H=0.02 \mathrm{~T}\right)$ amounts to $\xi=5.8(1)$. This corresponds to an average orientation of the particle moment with respect to the inducing magnetic field of $\langle\psi\rangle=\arccos (\mathscr{L}(\xi)) \sim 40^{\circ}$, which is in excellent agreement with the inclination angles observed for the domains of aligned particles.

The particle alignment within these oriented domains is comparable to what was found for self-organization in a static magnetic field, corresponding to a nematic phase with $\sim 5^{\circ}$ orientation distribution. This nearly collinear orientation is again attributed to packing constraints of the elongated particles with high aspect ratio. However, whereas orientation of the collinear nanospindle arrangement is induced by the planar substrate in the case of self-organization in a static magnetic field, the perpendicular orientation observed here is clearly induced by the rotating magnetic field. Both SEM and SAXS thus confirm the potential of rotating magnetic fields towards oriented arrangements of hematite spindles perpendicular to the substrate.

\subsection{Directional magnetic properties}

The oriented arrangement of hematite particles in different geometries introduced above allows for a detailed study of the directional magnetic properties of the assemblies in connection with the particle alignment. Due to the strong magnetocrystalline anisotropy in hematite, the spontaneous magnetization is sensitive to the orientation of the magnetic easy axis in the inducing field. ${ }^{\mathbf{1 7}}$ Maximum spontaneous magnetization is thus observed in the basal plane of bulk hematite single crystals (2000 $\mathrm{A} \mathrm{m}^{-1}$ (ref. 17 and 22)), whereas the corresponding spontaneous magnetization for a powdered hematite sample without preferred orientation is significantly lower $\left(\frac{\pi}{4} \sigma_{\mathrm{s}, \text { bulk }}=1550 \mathrm{~A} \mathrm{~m}^{-1}\right.$ (ref. 17)). In dilute dispersion, hematite nanoparticles are able to orient freely in the inducing magnetic field. The spontaneous magnetization measured in dispersion is thus regarded as the equivalent of measurements in the basal plane of a single crystal as opposed to a 3D disordered powder. As a reference for perfectly aligned spindles, we will thus refer to the spontaneous magnetization of $\sigma_{\mathrm{s}}=1187$ (3) $\mathrm{A} \mathrm{m}^{-1}$, determined in 3.1. Field dependent magnetization measurements in orthogonal directions of the studied spindle assemblies are presented in Fig. 5. All measurements exhibit the hysteretic behavior of arrested nanoparticles below the superparamagnetic blocking temperature. Observed coercive fields in the range of $\mu_{0} H=0.05 \mathrm{~T}$ are lower than those known for bulk hematite powder $\left(\mu_{0} H=0.33 \mathrm{~T}\right),{ }^{17}$ thus indicating a significant influence of superparamagnetic relaxation, which also explains the low spontaneous magnetization observed in our spindles. The hysteretic behavior originates in the weakly ferromagnetic magnetization contribution of hematite, whereas the excess susceptibility $\chi_{\text {exc }}$ at high applied fields is associated with the antiferromagnetic magnetization contribution. We observe an excess susceptibility in the range of $\chi_{\text {exc }}=590(35) \mathrm{A} \mathrm{m}^{-1} \mathrm{~T}^{-1}$, which is nearly constant for all samples and has been corrected for display in Fig. 5. The spontaneous magnetization $\sigma_{\mathrm{s}}$, associated with the weakly ferromagnetic magnetization contribution, is obtained by extrapolation of the linear part of the high-field magnetization to zero field. Magnetization measurements of the sample deposited without application of a directing magnetic field (Fig. 5a) are 


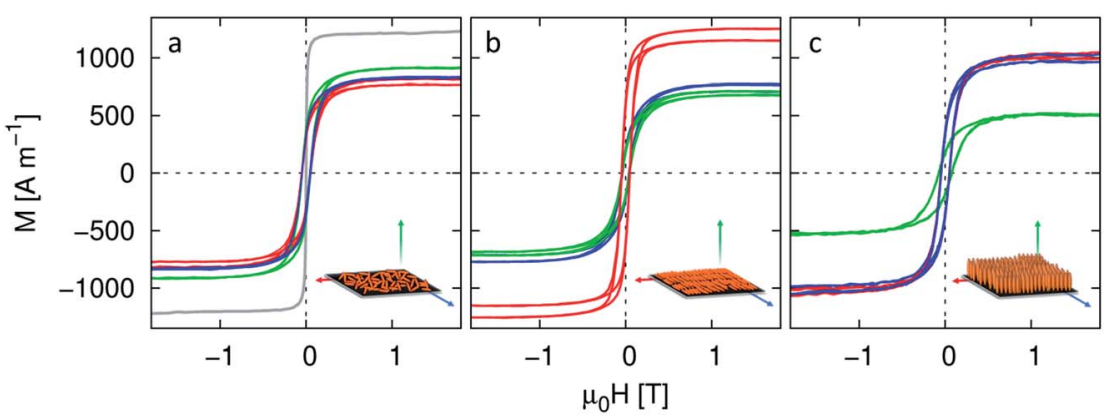

Fig. 5 Directionally resolved magnetization measurements of the discussed nanospindle assemblies as deposited (a) without applied field, compared with magnetization by particles in dispersion (grey), (b) with applied static field, and (c) with applied rotating field. Data have been corrected by $\chi_{\mathrm{exc}}$ at high field.

directionally equivalent, with a spontaneous magnetization of $\sigma_{\mathrm{s}}=840(50) \mathrm{A} \mathrm{m}^{-1}$, which is in excellent agreement with the expected spontaneous magnetization of the isotropic distribution as compared to perfectly aligned spindles. This confirms the isotropic nature of this sample, as well as the correct calibration of the magnetization measurements for the planar sample geometry. Deviations of the directional spontaneous magnetization in this sample amount to $50 \mathrm{~A} \mathrm{~m}^{-1}$ and are hence considered as the overall instrumental uncertainties. The average spontaneous magnetizations of the samples oriented in static and rotating magnetic fields of $\sigma_{\mathrm{s}}=880(90) \mathrm{A} \mathrm{m}^{-1}$ and $820(50) \mathrm{A} \mathrm{m}^{-1}$ are in agreement with the isotropic sample. However, a pronounced directional dependence is observed for these nearly collinear particle arrangements.

For the sample self-organized in a static magnetic field, a large spontaneous magnetization of $\sigma_{\mathrm{s}}=1190(20) \mathrm{A} \mathrm{m}^{-1}$, comparable to the perfectly aligned spindles in dispersion, is observed in the direction of the magnetic field during deposition, i.e. the global easy axis direction in the sample (Fig. 5b). The nearly identical magnetization curves with $\sigma_{\mathrm{s}}=725(50) \mathrm{A} \mathrm{m}^{-1}$, observed perpendicular to the easy axis, might be related to $2 \mathrm{D}$ disorder of the particle orientation perpendicular to the inducing field direction. However, SAXS results have proven collinear particle orientation, as opposed to 2D disorder throughout the sample on a global scale (3.2). In consequence, we reason that rotation of the magnetic moment out of the easy axis results in reduction of the spontaneous magnetization, which is identical within and outside the basal plane of the material. This is in agreement with recent observations of a uniaxial anisotropy within the hematite basal plane ${ }^{28}$ likely induced by enhanced magnetoelastic anisotropy, as compared to magnetocrystalline and shape anisotropies. ${ }^{17}$

Magnetization measurements by the sample organized in a rotating magnetic field (Fig. 5c) exhibit a pronounced anisotropy between directions parallel and perpendicular to the substrate, i.e. perpendicular and parallel to the rotation axis of the inducing magnetic field during self-organization. The lowest spontaneous magnetization of $\sigma_{\mathrm{s}}=435(10) \mathrm{A} \mathrm{m}^{-1}$ is found perpendicular to the substrate, i.e. parallel to the long particle axis, indicating a hard magnetic direction of the arrangement. The nearly identical magnetization measurements in the sample plane with $\sigma_{\mathrm{s}}=1012(40) \mathrm{A} \mathrm{m}^{-1}$ illustrate that the sample is not only structurally 
but also magnetically isotropic in these directions, i.e. the easy axis is evenly distributed in the plane. This is supported quantitatively by excellent agreement with the 2D average of $\sigma_{\mathrm{s}}\left(960(70) \mathrm{A} \mathrm{m}^{-1}\right.$ ) in the statically aligned sample (Fig. 5b). Isotropic distribution of particle easy axes in the substrate plane is a direct consequence of the deposition technique using a rotating magnetic field and the likely different time of arrest (and thus orientation of the easy axis) of the individual particles in the assembly. This also indicates that the inducing magnetic field of $\mu_{0} H=20 \mathrm{mT}$ is significantly stronger than any dipolar interparticle interaction. The magnetic dipolar interaction energy between neighboring nanospindles can be approximated as point dipoles according to

$$
E_{\text {dip }}=\frac{\mu_{0} \mu^{2}}{4 \pi d^{3}}
$$

and amounts with the particle moment of $\mu=1.19(1) \times 10^{-18} \mathrm{~J} \mathrm{~T}^{-1}$ and the interparticle correlation distance of $d=64(1) \mathrm{nm}$ to $E_{\mathrm{dip}}=5.4 \times 10^{-22} \mathrm{~J}$, which is well below both thermal $\left(E_{\mathrm{th}}=k_{\mathrm{B}} T=4.14 \times 10^{-21} \mathrm{~J}\right)$ and Zeeman $\left(E_{\mathrm{Z}}=-\mu \mu_{0} H=\right.$ $-2.4 \times 10^{-20} \mathrm{~J}$ ) energies in the studied conditions. Consequently, orientation of the nanospindles in the inducing magnetic field is the dominant effect.

Directionally resolved magnetization measurements have thus conclusively revealed a distinct orientation distribution of the easy axes in hematite nanospindle assemblies with structurally equally collinear particle orientation.

\section{Conclusion}

In the frame of this work, we have successfully exploited the anisotropic dynamic properties of elongated hematite particles for magnetic field-induced self-organization. Collinear, oriented arrangements of weakly ferromagnetic hematite spindles are achieved by the application of static as well as dynamic magnetic fields during self-organization. The collinear particle arrangement is attributed to a combination of the inducing magnetic field and steric effects. Horizontal orientation of the particle ensemble is induced by the combination of a static directing magnetic field and the planar geometry of a flat substrate, whereas vertical orientation of the particle ensemble is achieved by deposition in a rotating magnetic field of carefully adjusted rotation frequency. The obtained assemblies exhibit strongly anisotropic magnetic properties arising from the orientational particle alignment. For the statically aligned sample, orientation of the magnetic easy axis in the basal plane of the nanoparticles collinear with the applied field direction is confirmed. For the dynamically aligned sample, the magnetic easy axis is statistically distributed in the substrate plane. This is a direct result of the low dipolar interparticle interaction as compared to the inducing field strength, which inhibits alignment of the easy axes in the structurally collinearly arranged particle ensemble.

Our work thus demonstrates a promising approach towards the direction of both structural and collective magnetic anisotropy in nanostructured materials.

\section{Acknowledgements}

We would like to thank Ruth Bruker (Universität zu Köln) for SEM images. We gratefully acknowledge financial support from the Fonds der Chemischen 
Industrie as well as the UoC Key Profile Area III - Quantum Matter \& Materials. This work benefitted from SasView software, originally developed by the DANSE project under NSF award DMR-0520547.

\section{References}

1 G. M. Whitesides and B. Grzybowski, Science, 2002, 295, 2418-2421.

2 S. C. Glotzer and M. J. Solomon, Nat. Mater., 2007, 6, 557-562.

3 S. Polarz, Adv. Funct. Mater., 2011, 21, 3214-3230.

4 S. Sacanna, D. J. Pine and G.-R. Yi, Soft Matter, 2013, 9, 8096-8106.

5 A. Ahniyaz, Y. Sakamoto and L. Bergström, Proc. Natl. Acad. Sci. U. S. A., 2007, 104, 17570-17574.

6 S. Disch, E. Wetterskog, R. P. Hermann, G. Salazar-Alvarez, P. Busch, T. Brückel, L. Bergström and S. Kamali, Nano Lett., 2011, 11, 1651-1656.

7 J.-M. Meijer, F. Hagemans, L. Rossi, D. Byelow, S. I. R. Castillo, A. Snigirev, I. Snigireva, A. P. Philipse and A. V. Petukhov, Langmuir, 2012, 28, 7631-7638. 8 H. Cölfen and M. Antonietti, Angew. Chem., Int. Ed., 2005, 44, 5576-5591.

9 A. van Blaaderen, M. Dijkstra, R. van Roij, A. Imhof, M. Kamp, B. W. Kwaadgras, T. Vissers and B. Liu, Eur. Phys. J. Spec. Top., 2013, 222, 2895-2909.

10 A. B. G. M. Leferink op Reinink, E. van den Pol, G. J. Vroege and A. V. Petukhov, J. Colloid Interface Sci., 2014, 428, 316-320.

11 T. H. Besseling, M. Hermes, A. Fortini, M. Dijkstra, A. Imhof and A. van Blaaderen, Soft Matter, 2012, 8, 6931.

12 Q. Pankhurst, J. Connolly, S. Jones and J. Dobson, J. Phys. D: Appl. Phys., 2003, 36, R167-R181.

13 D. T. N. Chen, Q. Wen, P. A. Janmey, J. C. Crocker and A. G. Yodh, Annu. Rev. Condens. Matter Phys., 2010, 1, 301.

14 J. E. Martin and A. Snezhko, Rep. Prog. Phys., 2013, 76, 126601.

15 M. Ozaki, S. Kratohvil and E. Matijević, J. Colloid Interface Sci., 1984, 102, 146-151.

16 J. Wagner, T. Autenrieth and R. Hempelmann, J. Magn. Magn. Mater., 2002, 252, 4-6.

17 F. Bødker, M. F. Hansen, C. Bender Koch, K. Lefmann and S. Mørup, Phys. Rev. B: Condens. Matter, 2000, 61, 6826-6838.

18 M. Reufer, H. Dietsch, U. Gasser, A. Hirt, A. Menzel and P. Schurtenberger, J. Phys. Chem. B, 2010, 114, 4763-4769.

19 C. Märkert, B. Fischer and J. Wagner, J. Appl. Crystallogr., 2011, 44, 441-447.

20 J. Pedersen, Adv. Colloid Interface Sci., 1997, 70, 171-210.

21 A. Guinier, Ann. Phys., 1939, 12, 161.

22 P. J. Flanders and J. P. Remeika, Philos. Mag., 1965, 11, 1271-1288.

23 M. Reufer, H. Dietsch, U. Gasser, B. Grobety, A. Hirt, V. K. Malik and P. Schurtenberger, J. Phys.: Condens. Matter, 2011, 23, 065102.

24 F. Perrin, J. Phys.Radium, 1936, 7, 1-11.

25 A. Günther, P. Bender, A. Tschöpe and R. Birringer, J. Phys.: Condens. Matter, 2011, 23, 325103.

26 L. Onsager, Ann. N. Y. Acad. Sci., 1949, 51, 627.

27 T. Bigioni, X. Lin, T. Nguyen, E. Corwin, T. Witten and H. Jaeger, Nat. Mater., 2006, 5, 265-270.

28 L. Roeder, P. Bender, M. Kundt, A. Tschöpe and A. M. Schmidt, Phys. Chem. Chem. Phys., 2015, 17, 1290-1298. 